\title{
An identity-based ring signature scheme from bilinear pairings
}

\author{
Chih-Yin Lin and Tzong-Chen $\mathrm{Wu}^{*}$ \\ Institute of Information Management, National Chiao Tung University, Taiwan \\ Email: lincy@iim.nctu.edu.tw \\ *Department of Information Management, \\ National Taiwan University of Science and Technology, Taiwan \\ Email: tcwu@cs.ntust.edu.tw
}

\begin{abstract}
At the conference Asiacrypt 2001, Rivest, Shamir and Tauman firstly addressed the concept of ring signature. In this paper we propose an identity-based ring signature scheme from bilinear pairings. As compared with the Zhang-Kim scheme (presented at the conference Asiacrypt 2002), our scheme is more efficient in computation and requires fewer pairing operations.
\end{abstract}

Keywords: ring signature, identity-based, bilinear pairings.

\section{Introduction}

At the conference Asiacrypt 2001, Rivest, Shamir and Tauman [1] firstly addressed the concept of ring signature. A ring signature can be considered as a simplified group signature with no manager, no group setup procedure, and no revocation mechanism against signer's anonymity. In a ring signature scheme, the information of all possible signers, i.e. ring members, serves as a part of the ring signature for the signed message. A valid ring signature will convince the verifier that the signature is generated from one of the ring members, without revealing any information about which ring member is the actual signer. Herein, the anonymous property is referred to as signer-ambiguity. Applications of ring signatures include leaking secrets and authenticated communication [1].

Recently the bilinear pairings have been found advantageous in designing various cryptographic schemes, especially for those using identity-based public keys, e.g. the identity-based encryption [2] and the identity-based signature [3]. For all these schemes, the performance heavily depends on the cost of computing pairing operations. Therefore, many efforts such as [4-5] have been focused on speeding up the computation of pairings. Although significant results have been proposed [4-5], it is still important to design new schemes that require less pairing operations to achieve better performance.

In this paper, we will propose an identity-based ring signature scheme from bilinear pairings. As compared with the Zhang-Kim scheme ([6], presented at the conference Asiacrypt 2002), our scheme is computationally more efficient, especially for the pairing operations required during signature verification. In below, the bilinear pairing is introduced. Then, we will propose our scheme and give discussions and analysis.

\section{Bilinear pairing}

Assume $G_{1}$ is an additive cyclic group of prime order $q$; and, $G_{2}$ is a multiplicative cyclic group of prime order $q$. As in [2-6], $G_{1}$ can be considered as a subgroup of points on an elliptic curve over a finite field; and, $G_{2}$ a subgroup of the multiplicative group of a related finite field. The bilinear pairing $e: G_{1} \times G_{1} \rightarrow G_{2}$ has the following properties:

i) Bilinear: For all $P, Q \in G_{1}$ and $a, b \in Z_{q}^{*}, e(a P, b Q)=e(a b P, Q)=e(P, a b Q)=e(P, Q)^{a b}$.

ii) Non-degenerate: There exists a $P \in G_{1}$, such that $e(P, P) \neq 1$. 
iii) Computable: Given $P, Q \in G_{1}$, there is an efficient algorithm to compute $e(P, Q)$.

As specified in [2-6], the decisional Diffie-Hellman problem (DDHP) in $G_{1}$ should be easy. The DDHP in $G_{2}$, the computational Diffie-Hellman problem (CDHP) and the discrete logarithm problem (DLP) in both $G_{1}$ and $G_{2}$ should be hard. The inversion of the bilinear pairing should be hard, i.e. the bilinear pairing inversion problem (BPIP), which is defined as:

- BPIP: Given $P \in G_{1}$ and $e(P, Q) \in G_{2}$, find $Q \in G_{1}$.

As analyzed in [7], BPIP is reducible from CDHP in $G_{2}$ and can be reduced to DLP in $G_{2}$. Such bilinear pairing $e$ has been successfully realized on certain elliptic curves, such as the modified Weil pairing [2] and Tate pairing [4-5].

\section{Proposed scheme}

Assume there is a trusted key generation center (TKGC) that will establish the identity-based cryptosystem and generate private keys for users. Initially, TKGC selects $q, G_{1}, G_{2}$, and $e$, as defined in the previous section. Then, TKGC selects $P \in G_{1}$ as the generator of $G_{1}$ and defines one-way hash function $H_{1}:\{0,1\}^{*} \rightarrow G_{1}$ and $H_{2}:\{0,1\}^{*} \rightarrow Z_{q}$. TKGC's private key is $s \in Z_{q}^{*}$ and the public key is $P_{\text {pub }} \in G_{1}$, computed as:

$P_{\text {pub }}=s P$.

EQ.1

Finally, TKGC keeps $s$ secretly and publishes $\left\{G_{1}, G_{2}, q, e, P, P_{p u b}, H_{1}, H_{2}\right\}$. The proposed scheme consists of three phases: key generation, ring signature generation, and ring signature verification, stated as follows.

$<$ Key generation> For each signer $u_{i}$ in the system, TKGC generates $u_{i}$ 's private key $D_{i}$ based on $u_{i}$ 's unique identity $I D_{i}$, as:

$$
D_{i}=s H_{1}\left(I D_{i}\right) \text {. }
$$

EQ.2

$<$ Ring signature generation> Without loss of generality, assume there are $n$ members $u_{1}, u_{2}, \ldots, u_{n}$ in the ring chosen by the actual signer $u_{a}, 1 \leq a \leq n$. Given the message $m$ to be signed, $u_{a}$ firstly prepares a set $L$ of the unique identities from all ring members, i.e. $L=\left\{I D_{1}, I D_{2}, \ldots, I D_{n}\right\}$. Then, $u_{a}$ selects $A \in G_{1}$ at random and computes $c_{a+1}$ as:

$$
c_{a+1}=e(A, P) .
$$

In accordance with the order of $i=a+1, a+2, \ldots, a+(n-1)(\bmod n), u_{a}$ randomly selects $R_{i} \in G_{1}$, and computes $c_{i+1}$ as:

$$
c_{i+1}=e\left(P_{p u b}, c_{i} H_{1}\left(I D_{i}\right)\right)^{H_{2}(m \| L)} \cdot e\left(R_{i}, P\right) .
$$

Let $R_{n}=R_{0}$. Then, $u_{a}$ computes $R_{a}$ and $R$ as:

$R_{a}=A-H_{2}(m \| L) c_{a} D_{a}$,

$R=\sum_{i=1}^{n} R_{i}$.

EQ.6

Finally, let $c_{n}=c_{0} .\left(c_{1}, c_{2}, \ldots, c_{n}, R\right)$ is the ring signature for message $m$ with ring members specified by $L$.

$<$ Ring signature verification> Given message $m$, its ring signature $\left(c_{1}, c_{2}, \ldots, c_{n}, R\right)$, and the set $L$ of identities of all ring members, the verifier can check the validity of the signature by testing if:

$$
\prod_{i=1}^{n} c_{i}=e\left(P_{p u b}, \sum_{i=1}^{n} c_{i} H_{1}\left(I D_{i}\right)\right)^{H_{2}(m \| L)} \cdot e(R, P) .
$$

EQ.7

\section{Security analysis}

We consider three security requirements to the proposed scheme: key secrecy, unforgeability and 
signer-ambiguity. In below, we will give their definitions and show that the proposed scheme achieves them under the intractability of DLP and BPIP.

- Key secrecy: Given all public information, deducing signer $u_{i}$ 's private key $D_{i}$ is computationally infeasible.

- Unforgeability: Given a message $m$, a set $L$ of identities of ring members, and all public information, any $u_{k} \notin L$ cannot compute a valid ring signature that satisfies EQ.7.

- Signer-ambiguity: Given message $m$, its ring signature $\left(c_{1}, c_{2}, \ldots, c_{n}, R\right)$, and the set of ring members $L=\left\{I D_{1}, I D_{2}, \ldots, I D_{n}\right\}$, it is unconditionally infeasible to find out who is the actual signer.

<Achievement of key secrecy> In the proposed scheme, all signers' private keys are generated by EQ.2. Thus, computing $u_{i}$ 's private key $D_{i}$ from the corresponding public key $H_{1}\left(I D_{i}\right)$ requires the knowledge of TKGC's private key $s$. As defined in EQ.1, $s$ is protected under the intractability of DLP in $G_{1}$. Moreover, if the adversary assumes $u_{a}, 1 \leq a \leq n$, is the actual signer for ring signature $\left\{L=\left\{I D_{1}, I D_{2}, \ldots, I D_{n}\right\},\left(c_{1}, c_{2}, \ldots, c_{n}, R\right), m\right\}$ and intends to compute $u_{a}$ 's private key $D_{a}$ from EQ.5. The adversary will have to know $A$ for such attack. By EQ.3, he will face the intractability of BPIP to obtain $A$. Therefore, due to DLP in $G_{1}$ and BPIP, key secrecy is assured.

<Achievement of unforgeability> Given the set $L=\left\{I D_{1}, I D_{2}, \ldots, I D_{n}\right\}$ of all ring members, an adversary $u_{k} \notin L$ may try to compute a valid ring signature for message $m$ via two ways. First, he randomly chooses the values of $\left(c_{1}, c_{2}, \ldots, c_{n}\right)$ and tries to compute $R$ such that $\left(c_{1}, c_{2}, \ldots, c_{n}, R\right)$ will satisfy EQ.7. Computing such $R$ is equivalent to solving it in $e(R, P)=\alpha$, where $\alpha=\left(\prod_{i=1}^{n} c_{i}\right) \cdot e\left(P_{p u b}, \sum_{i=1}^{n} c_{i} H_{1}\left(I D_{i}\right)\right)^{-H_{2}(m \| L)}$. This will lead to the BPIP intractability. Second, w.l.o.g the adversary chooses $\left(c_{2}, \ldots, c_{n}, R\right)$ at random and then tries to compute $c_{1}$ such that $\left(c_{1}, c_{2}, \ldots, c_{n}, R\right)$ will satisfy EQ.7. Computing such $c_{1}$ is equivalent to solving it in $c_{1}=y \cdot z^{c_{1}}$, where $y=\left(\prod_{i=2}^{n} c_{i}\right)^{-1} \cdot e\left(P_{p u b}, \sum_{i=2}^{n} c_{i} H_{1}\left(I D_{i}\right)\right)^{H_{2}(m \| L)} \cdot e(R, P)$ and $z=e\left(P_{p u b}, H_{1}\left(I D_{1}\right)\right)^{H_{2}(m \| L)}$, which as a result is the intractable DLP in $G_{2}$. Therefore, due to DLP in $G_{2}$ and BPIP, unforgeability is achieved.

$<$ Achievement of signer-ambiguity> In a valid ring signature $\left(c_{1}, c_{2}, \ldots, c_{n}, R\right)$ with $L=\left\{I D_{1}, I D_{2}, \ldots, I D_{n}\right\}$ generated by $u_{a}, 1 \leq a \leq n$, all $c_{i}$ 's, $i \neq a+1$, are computed by EQ.4. Since $R_{i} \in G_{1}$ is chosen uniformly at random, each $c_{i}$ is uniformly distributed over $G_{2}$, for all $i \neq a$. As for the starting point of the ring, $c_{a}$ is computed by EQ.3. Because $A$ is also chosen uniformly at random, $c_{a}$ is uniformly distributed over $G_{2}$. Thus, regardless who the actual signer is and how many ring members involved, $\left(c_{1}, c_{2}, \ldots, c_{n}\right)$ biases to no specific ring member.

\section{Efficiency}

The computational costs required are considered in terms of the notations: $P a$ the pairing, $M u G_{1}$ the multiplication in $G_{1}, A d G_{1}$ the addition in $G_{1}, M u G_{2}$ the multiplication in $G_{2}$, and $\mathrm{Ha}$ the hash function computation. Notice that the cost for computing an exponentiation in $G_{2}$ is evaluated herein as a multiplication in $G_{1}$ and we ignore the cost of computing $H_{1}\left(I D_{i}\right)$.

Table 1 shows the costs in ring signature generation and verification of the proposed scheme, and the comparison with Zhang-Kim's scheme [6]. We also consider possible pre-computations for signature generation that are indirectly relevant to the message signing, e.g. EQ 3. and EQ.6.

Consequently, we can see that the proposed scheme has comparable performance with Zhang-Kim's scheme for signature generation. As for signature verification, the proposed scheme requires only two pairing computations while Zhang-Kim needs $2 n$. If we consider the complexity instead of the exact number of operations as seen in Table 1, the comparison is more significant. For 
signature generation, $\mathrm{Pa}, M u G_{1}$ and $M u G_{2}$ are $\mathrm{O}(n)$, and $H a$ and $A d G_{1}$ are $\mathrm{O}(1)$ in our scheme, while these measures are all $\mathrm{O}(n)$ in [6]. For signature verification, $P a$ and $H a$ are $\mathrm{O}(1)$ in our scheme, while they are $\mathrm{O}(n)$ in [6].

Table 1 -

Computational costs for the proposed scheme and the Zhang-Kim scheme ( $\boldsymbol{n}$ ring members)

\begin{tabular}{cccc}
\hline \hline & Signature generation & $\begin{array}{c}\text { Signature generation } \\
\text { (with pre-computation) }\end{array}$ & Verification \\
\hline $\begin{array}{c}\text { Proposed } \\
\text { scheme }\end{array}$ & $\begin{array}{c}(2 \boldsymbol{n}-1) P a+H a+ \\
\text { Ad } G_{1}+(2 \boldsymbol{n}-2) M u G_{1} \\
+\boldsymbol{n} M u G_{2}\end{array}$ & $\begin{array}{c}(\boldsymbol{n}-1) P a+H a+A d G_{1}+ \\
(2 \boldsymbol{n}-2) M u G_{1}+\boldsymbol{n} M u G_{2}\end{array}$ & $\begin{array}{c}2 P a+H a+(\boldsymbol{n}-1) A d G_{1}+ \\
(\boldsymbol{n}+1) M u G_{1}+\boldsymbol{n} M u G_{2}\end{array}$ \\
\hline \multirow{2}{*}{$\begin{array}{c}(2 \boldsymbol{n}-1) P a+\boldsymbol{n} H a \\
+\boldsymbol{n} A d G_{1}+\boldsymbol{n} M u G_{1}\end{array}$} & $\boldsymbol{n} P a+\boldsymbol{n} H a+\boldsymbol{n} A d G_{1}+$ & $2 \boldsymbol{n P a + n H a +}$ \\
& $+(\boldsymbol{n}-1) M u G_{2}$ & $\boldsymbol{n} M u G_{1}+(\boldsymbol{n}-1) M u G_{2}$ & $\boldsymbol{n} M u G_{1}+\boldsymbol{n} M u G_{2}$ \\
\hline \hline
\end{tabular}

\section{Conclusions}

In this paper, we have proposed a new ID-based ring signature scheme from bilinear pairings. The proposed scheme is more efficient as compared with the Zhang-Kim scheme, especially for the pairing operations required in signature generation. For the applications of ring signatures, such as authenticated communication, the proposed scheme is more suitable for those verifiers who only have limited computing power.

\section{References}

1. RIVEST, R., SHAMIR, A., and TAUMAN, Y.: 'How to leak a secret', Advances in Cryptology Asiacrypt 2001, LNCS 2248, Springer-Verlag, 2001, pp. 552-565.

2. BONEH, D., and FRANKLIN, M.: 'Identity-based encryption from the Weil pairing', Advances in Cryptology - Crypto 2001, LNCS 2139, Springer-Verlag, 2001, pp. 213-229.

3. PATERSON, K. G.: 'An identity-based signature from pairings on elliptic curves', Electronics Letters, 2002, 38(2), pp. 1025-1026.

4. BARRETO, P. S. L. M., KIM, H. Y., LYNN, B., and SCOTT, M.: 'Efficient Algorithms for Pairing-Based Cryptosystems', Advances in Cryptology - Crypto 2002, LNCS 2442, Springer-Verlag, 2002, pp. 354-368.

5. GALBRAITH, S. D., HARRISON, K., and SOLDERA, D.: 'Implementing the Tate pairing', Algorithmic Number Theory Symposium - ANTS-V, LNCS 2369, Springer-Verlag, 2002, pp. 324-337.

6. ZHANG, F., and KIM, K.: 'ID-based blind signature and ring signature from pairings', Advances in Cryptology - Asiacrypt 2002, LNCS 2501, Springer-Verlag, 2002, pp. 533-547.

7. YACOBI, Y.: 'A note on the bi-linear Diffie-Hellman assumption', IACR Cryptology ePrint Archive, Report 2002/113, 2002. 